\title{
Unresolving Pneumonia with Pleural effusion: Pulmonary Paragonimiasis
}

\author{
Deepshikha Gaire, 'Santosh Sharma, ${ }^{1}$ Kumar Poudel,' Pankaj Pant ${ }^{1}$ \\ 'Department of Internal Medicine, Institute of Medicine, Kathmandu, Nepal.
}

\begin{abstract}
Paragonimiasis is a zoonosis caused by many species of Paragonimus commonly P. westermani. Human get infected by eating raw, salted, pickled, smoked, partially cooked crustaceans (crayfish or crabs). Clinical manifestations ranges from non-specific symptoms like pain abdomen, diarrhea, urticarial rashes, fever to pleuropulmonary symptoms like cough, hemoptysis, chest pain and dyspnea. A 48 years, female presented at TUTH emergency with fever on and off for nine months, cough and shortness of breath for three months, lethargy, malaise and urticaria with history of raw crab intake one month prior to the onset of symptoms. Blood and pleural fluid analysis revealed raised total counts with eosinophilia and x-ray showed bilateral infiltration of lower lobes with pleural effusion. Diagnosis was confirmed by microscopic examination of sputum for Paragonimus. She responded well to Praziquantel. Pulmonary paragonimiasis must be considered in the differential diagnosis of unresolving pneumonia and unexplained hypereosinophilia.
\end{abstract}

Keywords: hypereosinophilia; paragonimiasis; pleural effusion; praziquantel.

\section{INTRODUCTION}

Paragonimiasis is a food-borne zoonotic disease caused by number of species of trematodes, Paragonimus commonly with $P$. westermani. It is a pulmonary disease, prevalent in Southeast Asia and Far East and has been estimated that 22 million people are affected globally., Human being become infected after eating raw or poorly cooked freshwater crabs or crayfish that have been encysted with the metacerciaria (embroyonated egg, infective stage). Thus, life cycle of paragonimus complete in three hosts, snail-crustaceans and human or animals. Clinical features are similar to Pneumonia and Pulmonary TB so many cases are misdiagnosed. A case of pulmonary paragonimiasis misdiagnosed as a pneumonia is presented herewith.

\section{CASE REPORT}

A 48 years old, female from Gulmi District of Nepal presented at TUTH emergency with fever and productive cough for nine months. Fever was on and off, high grade, intermittent type, associated with chills but no rigor and relieved by medications for few hours. Cough was productive, moderate in amount, mucoid, nonblood stained and nonfoul smelling. Initially she sought medical advice in a local private clinic where she was investigated, informed to have abnormal blood investigations (high total count with eosinophil predominent) and managed conservatively with oral medication which resolved her symptoms. However, after three months, she again developed high grade fever and cough. She also had history of lethargy, malaise and urticarial rashes but she denied chest pain, palpitation, burning micturation, joint pain, anorexia and weight loss. There was no significant known food or drug allergy. She had history of intake of raw crabs 1

Correspondence: Dr. Deepshikha Gaire, Institute of Medicine, Maharajguni, Kathmandu, Nepal. Email: dipc62@gmail.com, Phone: +977-9849070441. 
month prior to the onset of symptoms. With all these history she came to our center.

On examination, she was febrile with temperature of $101.4^{\circ} \mathrm{F}$ with stable other vital parameters maintaining oxygen saturation in room air. On chest examination stony dull percussion note was found on bilateral infrascapular and infra-axillary region and auscultation revealed decrease intensity of breath sound over same areas of chest.

Routine blood investigations revealed total count of 18,500 with eosionophil of $58 \%$ and raised ESR. Peripheral blood smear showed normocytic normochromia with eosinophilia. Rest of blood investigations were within normal limit. Chest $x$ ray showed bilateral lower lobe infiltration with pleural effusion. Bone marrow examination revealed bone marrow eosinophilia. Sputum smear were negative for AFB, fungi, pyogenic organism and malignant cells. Sputum microscopic examination for paragonimus showed operculated egg of paragonimus spp. Routine Stool examination was normal.

So on the background of history of intake of raw crabs, with symptoms of fever and cough, eosinophilia on investigation and ova of paragonimus seen in microscopic examination of sputum, diagnosis was confirmed as pulmonary paragonimiasis. She was treated with $25 \mathrm{mg} / \mathrm{kg}$ of Praziquantel three times a day for 3 days, responded well to treatment and was recovered fully on subsequent follow up.

\section{DISCUSSION}

Paragonimiasis, a parasitic zoonosis with pleuropulmonary and relatively infrequent extrapulmonary manifestation, is a subacute to chronic inflammatory condition. It commonly affects lung and is important cause of pulmonary disease worldwide especially in Asia, West-central Africa and Central and South America. ${ }^{3}$ It has vast global range, more than 40 species have been identified throughout world which infects domestic, wild animals (dogs, cats, bear, skunk, pigs etc.) and human beings. ${ }^{4}$ Most common species is Paragonimus westermani.

The life cycle of Paragonimus get completed in snailcrustaceans-human host. Human being get infected by eating uncooked, undercooked, pickled, marinated crayfish or crabs, which posses viable metacercarciae (infective stage) of Paragonimus. There is no route for human to human transmission. Metacercariae hatch in intestine, young worm penetrate the intestinal wall, peritoneum, then diaphragm, pleural cavity and finally reach lung where they live in pair and lay eggs. The unembroyonated egg eventually erode the bronchial wall and lead to cough and expectoration of sputum laden with eggs or if swallowed passed through the stool. In external environment eggs become embroyonated and miracidia hatches and seek the first intermediate host, snail and penetrate soft tissues. Miracidia undergo several stages of development inside snail where many cercariae emerge from snail. The cercariae invade second intermediate host, a crustacean such as crab or cray fish, where they encyst becoming metacercariae which is the infective stage for human.

Clinical presentation of this disease is due to the migration of larva from intestine to peritoneum, diaphragm and pleura then to lungs. In early stage patient may have elusive clinical picture or be asymptomatic or scarcely symptomatic. As in our case, patient became symptomatic only after few weeks of intake of raw crabs. In acute stage patient may present with diarrhea, pain abdomen, fever, malaise, night sweat etc. Late manifestations include fever, cough, hemoptysis, pleuritic chest pain, anorexia, weight loss, shortness of breath. Some time worm may migrate to brain to cause severe symptoms like headache, seizure, even vision loss.

However most common symptoms are productive cough with bloody sputum $(77.9 \%)$, nonproductive cough $(1.5 \%)$ and chest pain $(67.6 \%) .{ }^{5}$ To the contrary our patient had only non blood stained productive cough delaying the definitive diagnosis. Patient was investigated in line of Pneumonia and treated for the same but she didn't respond to treatment and develop recurrent pneumonia with effusion with eosinophilic predominent. Patient was investigated suspecting hematological malignancy as the blood investigation showed hypereosinophilia, however bone marrow examination showed normal findings except for eosinophilia. Paragonimiasis may be misdiagnosed as unresolving pneumonia, PTB, hypereosinophilic syndrome, lung cancer etc. especially in the areas where the condition is not so prevalent. Our case was initially diagnosed as hypereosinophilic syndrome, having hypereosinophilia for more than 6 months, feature of organs involvement (infiltration in lungs with effusion) and without any obvious cause for eosinophilia, so she was treated with steroids. Likewise, many studies has shown as paragonimiasis is misdiagnosed as PTB and treated with ATT. ${ }^{6}$ Sometimes patient may present with complication like corpulmonale, empyema. ${ }^{7,8}$

The radiological features ranges from fine transient infiltrates to cavities, cysts, calcified nodules and effusion making differentiation from Pneumonia, PTB and lung cancer difficult. As in our case chest $x$-ray showed bilateral lower lobe infiltration with effusion which can be easily misdiagnosed with aforementioned 
conditions. However, eosinophilia in blood and pleural fluid was supportive for diagnosis which was confirmed by visualization of opercualted, oval, yellowishcoloured eggs in sputum examination. Diagnosis of Paragonimiasis is confirmed by viewing the operculated eggs or parasites on tissue section or sputum or stool microscopic examination, although eggs typically are not presented until 2-3 months after infection. Detection of antibody to paragonimus using ELISA is more accurate in early infection, with a sensitivity of $92 \%$ and specificity of $97 \% .^{9}$

Praziquantel and triclabendazole are two drugs recommended by WHO to treat this disease. Praziquantel $25 \mathrm{mg} / \mathrm{kg}$ three times a day for three days is the most commonly used drug and has cure rate of $80-90 \% .{ }^{10}$ Patient was treated with the same regime and her sign and symptoms resolved in few days.

In Conculsion, clinical features of paragonimiasis may mimic unresolving pneumonia, so it should be suspected in a case not responding to treatment. This case report also highlights the importance of taking good personal history especially history of intake of raw cray-fish or crab. In this case patient was underdiagnosed for nine months, had multiple admissions, and was not responsive to standard treatment of pneumonia till the definitive diagnosis. So paragonimus must be suspected in cases of unresolving pneumonia in a country like Nepal where there are many crab eating communities so that, unwanted hospital admissions, investigations and unnecessary treatments could be avoided.

\section{Conflict of Interest: None.}

Consent: JNMA Case Report Consent Form was signed by the patient and the original is attached with the patient chart.

\section{REFERENCES}

1. Cabrera BD: Paragonimiasis in the Philippines: Current status. Arzneimittelforschung 34:1188, 1984. [PubMed]

2. Haswell-Elkins MR, Elkins DB. Lung and liver flukes. In: Leslie C, Albert B, Max S, ed Topley and Wilson's Microbiology and Microbial Infections; 9 th edn, Vol. V. New York: Oxford University Press; 1998;507-20. [Full Text]

3. Procop GW, Marty AM, Scheck DN, Mease DR etal. North American Paragonimiasis: a case report. Acta cytol. 2000 Jan-Feb;44(1),75-80. [PubMed]

4. Yokogawa M (1965). Paragonimus and paragonimiasis. Adv parasitol. 1969;7:375-87. [PubMed]

5. Wiwanitkit V. A summary of respiratory symptoms in Thai cases with pulmonary paragonimiasis. Internet J infect Dis. 2004;4:1. [Full Text]
6. Singh TS, Sugimaya H, Umehata A, Hiese S, Khalo K. Paragonimus heterotremus infection in Nagaland: A new focus of paragonimiasis in India. Indian J Med. Microbial. 2009 Apr-Jun;27(2):123-7. [Full Text]

7. CJ Chinwe, CO Cejatan. Paragonimiasis: an unusual case of corpulmonale; a case report. Respi Med. CME 2011;4(3):136-7. [DOI]

8. Defrain M, Hooker R. North American Paragonimiasis- case of severe clinical infection. chest April 2002;121(4):1368-72. [PubMed]

9. Singh TS, Sugiama H, Rangsiruji A. Paragonimus and paragonimiasis in India. Indian J med Res 2012 Aug;136(2):192-204. [PMC]

10. Liu Q, Wei F, Liu W, Yang S, Zhang X. Pargonimiasis:an important food- borne zoonosis in china. Trends parasitol. 2008 Jul;24(7):318-23. [PubMed] 